\title{
Shear Layer Driven Acoustic Modes in a Cylindrical Cavity
}

\author{
David B. Stephens,* \\ NASA Glenn Research Center, Cleveland, OH, 44070, USA. \\ Francisco R. Verdugo, ${ }^{\dagger}$ \\ Universita degli Studi Roma Tre, Italy. \\ Gareth J. Bennett $\ddagger$ \\ Trinity College Dublin, Dublin 2, Ireland.
}

16th AIAA/CEAS Conference (Stockholm, Sweden 2010)

This paper describes the interior acoustic pressure of a cylindrical cavity driven by a shear layer. Existing cavity flow literature is generally focused on rectangular cavities, where the resonance is either longitudinal or depth modes inside the cavity. The design of the present circular cavity is such that azimuthal duct modes can be excited in various combinations with depth modes depending on free stream velocity. An acoustic simulation of the system was used to identify the modes as a function of frequency when the system is driven by an acoustic point source. With appropriate manipulation of the free stream flow, abrupt mode switching and mode oscillation were both observed, and a condition with a dominant azimuthal mode was found. The strength of the lock-on was documented for the various resonance conditions, and the effects of the cavity opening size and location were studied.

$A \quad$ Area of cavity opening, $\mathrm{m}^{2}$

$c \quad$ Speed of sound, $\mathrm{m} / \mathrm{s}$

$f$ Frequency, $\mathrm{Hz}$

$H$ Height of cavity, mm

He Helmholtz number, $\frac{2 \pi f R}{c}$

$L \quad$ Length of cavity opening, $\mathrm{mm}$

$M \quad$ Mach number

$R \quad$ Cavity radius, $\mathrm{mm}$

$U \quad$ Free stream velocity, $\mathrm{m} / \mathrm{s}$

$W \quad$ Width of wind tunnel test section, $\mathrm{mm}$

$\Delta \quad$ Distance of opening from downstream edge of cavity

\section{Introduction}

Low Mach number cavity flows exist in numerous engineering applications. These systems are often susceptible to resonance based on feedback between the internal cavity pressure and the shear layer over the cavity opening. This phenomenon is important to understand because the unsteady pressure fluctuations may be very large, and can result in undesirable noise or damage to the cavity structure or equipment inside the cavity. These flows have been studied by a large number of researchers, including a few mentioned here

*Aerospace Engineer, Acoustics Branch B54-MS3, 21000 Brookpark Road, Cleveland, OH, 44070, USA. AIAA Member.

$\dagger$ Ph.D Student, Dipartimento di Ingegneria Meccanica Industriale, AIAA Student Member.

${ }_{\ddagger}^{\ddagger}$ Lecturer, Dept. of Mechanical and Manufacturing Engineering, Trinity College Dublin, Dublin 2, Ireland. AIAA Member. 
to illustrate the wide range of applications. A recent study by Langtry and Spalart ${ }^{1}$ used computational methods to predict the unsteady pressure inside a landing gear wheel well on a commercial aircraft geometry. Balasubramanian et al. ${ }^{2}$ have recently considered "sunroof buffeting" on a simplified vehicle geometry. Nakiboglu et al. ${ }^{3}$ have studied a series of sidebranch resonators as a model for flow in a corrugated pipe. Aly and Ziada ${ }^{4}$ have studied higher order modes in a pipe system with a sudden diameter change, and investigated the effect of cavity depth on acoustic particle velocity.

Shear layer driven cavity flows can exhibit several types of features generally described as resonance. Rockwell and Naudascher ${ }^{5}$ provided an extensive review, and categorized these types of systems. A brief summary will be provided here. The well-known example of high Mach number $(M>0.5)$ flow over a shallow cavity is known as a Rossiter ${ }^{6}$ cavity. In this system, the feedback mechanism is an upstream-traveling acoustic wave generated by turbulent structures hitting the downstream edge of the cavity. These acoustic waves have a wavelength close to the opening length. Resonance occurs if this acoustic frequency excites the shear layer oscillation. This type of feedback mechanism is not expected to lead to cavity resonance in the current system. The cavity discussed in this paper has an opening length that was varied between 40 and $50 \mathrm{~mm}$, corresponding to an acoustic frequency of around $8500 \mathrm{~Hz}$. This frequency is much higher than the frequency the the shear layer can oscillate at, given the low Mach number flow $(M<0.15)$ considered in the current paper.

Shear layer cavity flows can also excite standing acoustic waves in the cross-stream direction. These waves are reflected from the bottom of the cavity, and can feed back to the shear layer. These standing waves reflect off of the end of the cavity and any of: 1) the top of a cavity that is closed except for a small opening, 2) the opposite side of the wind tunnel, if the experiment is performed in a closed section, 3) between the ends of two opposing cavities in the case of a 'coaxial' resonator configuration, or 4) between the bottom of the cavity and the change of impedance due to the opening. Depth modes were studied by Yang et al., ${ }^{7}$ who specifically analyzed the effect of the streamwise dimension of the cavity. A coaxial sidebranch configuration was studied by Oshkai and Yan. ${ }^{8}$

Aside from standing acoustic waves, a shear layer driven cavity can act as a Helmholtz resonator (HR), where the oscillation is caused by compression of the volume of fluid inside the cavity. The preferred resonance frequency of this kind of system can be calculated as,

$$
f_{H R}=\frac{c}{2 \pi} \sqrt{\frac{A}{V l_{s}}}
$$

where $A$ is the area of the cavity opening, $V$ is its volume and $l_{s}$ is the length of the slug of air that oscillates in the opening, or neck of the cavity. For the cavity under consideration, the neck length is essentially zero, so an "effective" neck length equal to the opening length $\left(l_{s}=L\right)$ was used, and was found to match the experimental data well. Kook and Mongeau ${ }^{9}$ and Ma et al. ${ }^{10}$ have both studied Helmholtz resonators and accurately predicted the magnitude of the interior cavity pressure, when adequate information about the shear layer is available.

To the knowledge of the present authors, there has been no intentional attempt to study higher order acoustic modes in a cylindrical cavity excited by an external shear layer. The thorough understanding of resonance phenomena is important for designing systems to mitigate undesirable resonance. Results measured from the present experiment show that azimuthal modes can resonate in a cylindrical cavity with similar magnitudes to depth modes. A major contribution of this project will be the investigation of excitation of azimuthal cavity modes and the switching between the various types of acoustic standing waves in a cylindrical cavity. The following section discusses a simple experiment designed and instrumented for this project. Section III describes a computational simulation of the acoustics of the experimental domain. Section IV presents selected results from the experiment and Section V provides a summary of the findings.

\section{Experiment}

A cavity resonance experiment that accommodates a variety of the feedback mechanisms described in Section I has been designed and constructed. Operating the experiment over a range of flow speeds provides a method to switch between the various resonance conditions. A schematic of the current experiment is given as Figure 1. The experiment consists of a $125 \mathrm{~mm}$ square wind-tunnel test section, with an attached cylindrical cavity of $R=119 \mathrm{~mm}$ internal radius and a $D=493 \mathrm{~mm}$ depth. The large radius of the cylinder was selected in order to accommodate higher-order azimuthal acoustics modes at frequencies low enough to 
be excited by a low Mach number shear layer. The first azimuthal mode was designed to cut on at $837 \mathrm{~Hz}$, and the second at $1387 \mathrm{~Hz}$. A series of rectangular openings with sharp leading and trailing edges the cavity to the wind tunnel. The diameter of the cavity is significantly larger than the width of the wind tunnel test section, so end plates were needed on both sides of the cavity. The cavity was sealed except for the opening to the wind tunnel.
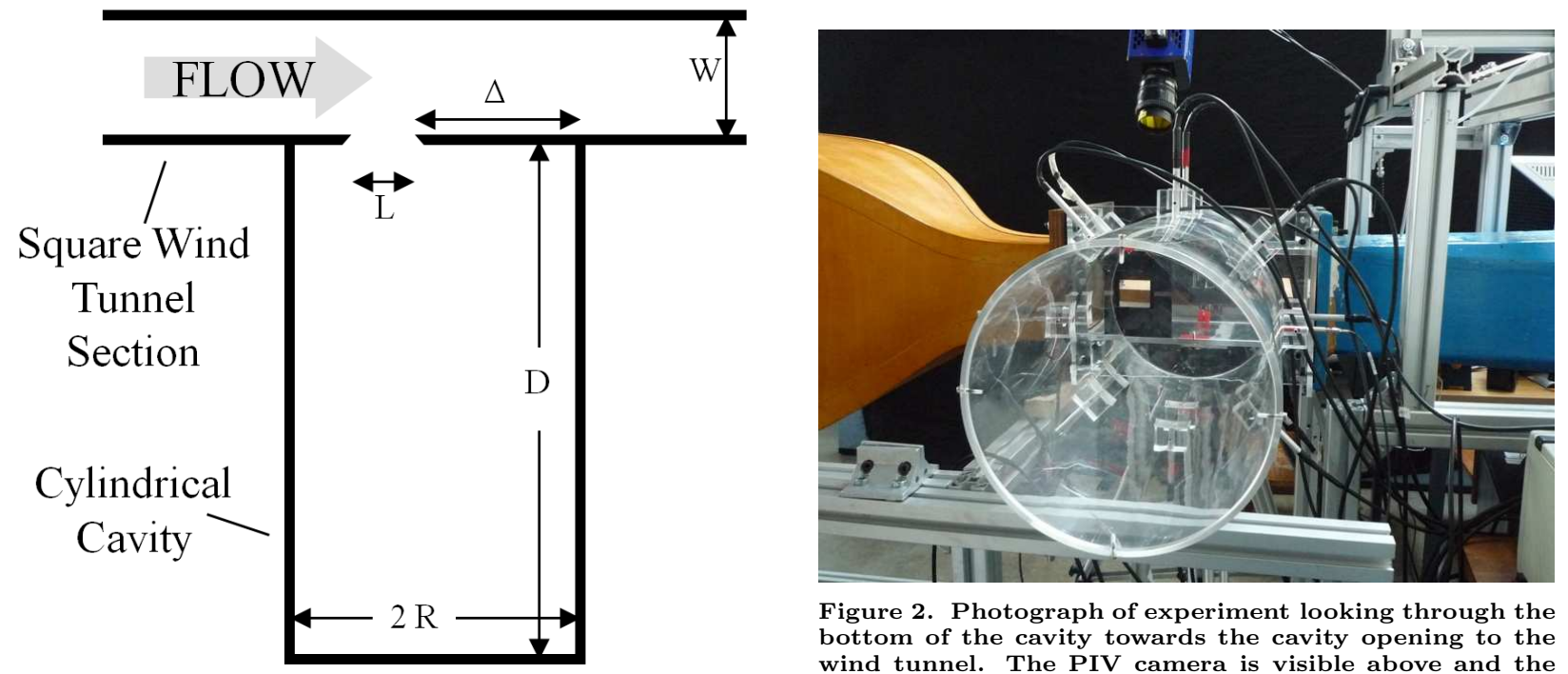

Figure 2. Photograph of experiment looking through the bottom of the cavity towards the cavity opening to the wind tunnel. The PIV camera is visible above and the cavity is instrumented with two rings of microphones.

Figure 1. Schematic of cavity experiment. Not to scale.

The focus of the results presented in this report describe the effect of the length and location of the cavity opening, defined by the parameters $L$ and $\Delta$. All of the openings considered in this report were rectangular with a width of $40 \mathrm{~mm}$. The $L=40 \mathrm{~mm}$ opening could be positioned at the center of the cavity $(\Delta=100)$, at $R / 2(\Delta=40)$ and at the edge of the cavity $(\Delta=0)$. At the edge of the cavity, openings with $L=40,45$, and $50 \mathrm{~mm}$ were tested. The combinations presented in this paper are summarized in Table 1.

\begin{tabular}{|c|c|c|c|c|c|}
\hline$L(\mathrm{~mm})$ & 40 & 45 & 50 & 40 & 40 \\
\hline$\Delta(\mathrm{mm})$ & 0 & 0 & 0 & 40 & 100 \\
\hline
\end{tabular}

Table 1. Cavity opening dimensions and locations

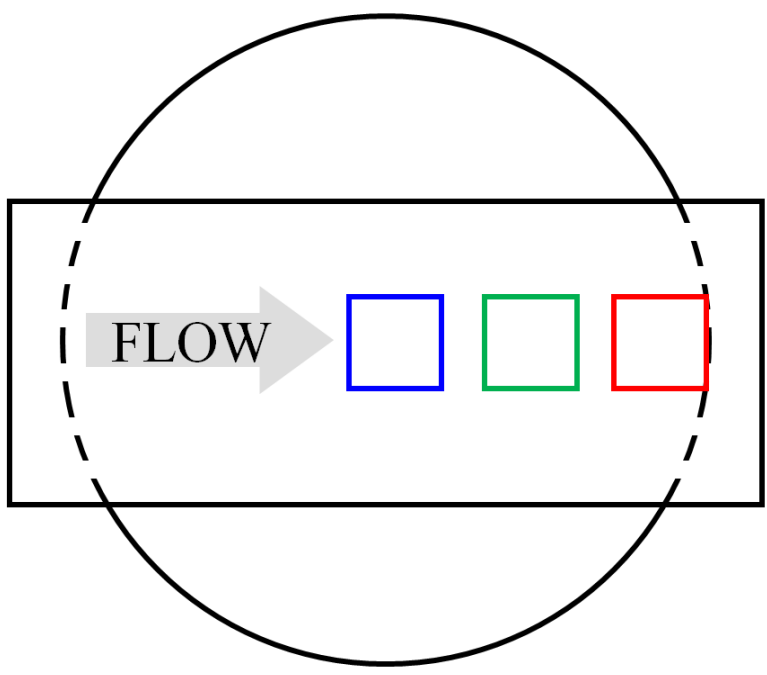

Figure 3. Sketch showing variation in cavity opening location. Not to scale.

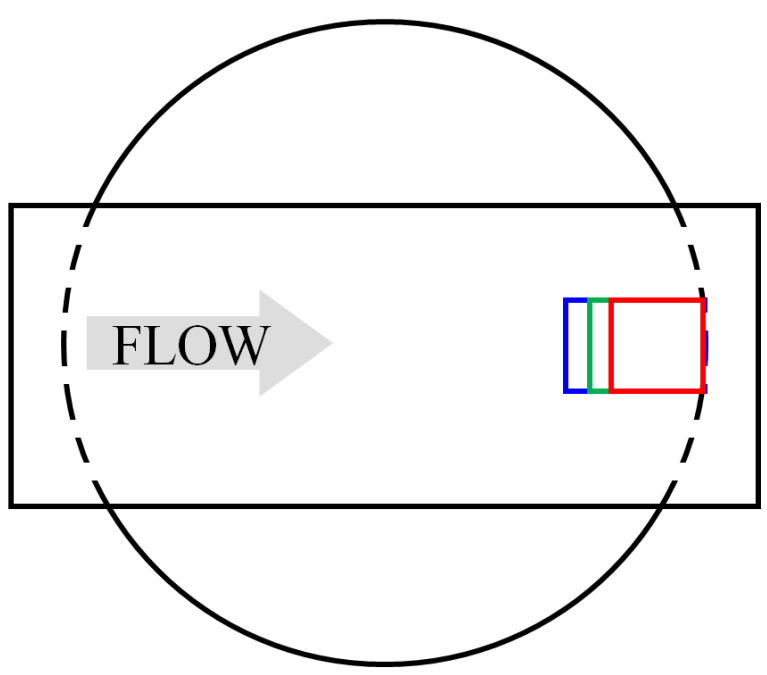

Figure 4. Sketch showing variation in cavity opening lengths. Not to scale.

The shear layer excitation frequency can be estimated using the empirical relationship suggested by 
Rossiter. ${ }^{6}$ As mentioned in the introduction, the upstream-propagating acoustic feedback mechanism usually associated with Rossiter is not expected to occur in the present experiment. This is because the acoustic wavelength corresponding to the cavity opening length would be of the order of $8 \mathrm{kHz}$, far higher than any expected shear layer oscillation frequency based on the velocity of the tunnel. Rossiter's equation for the shear layer excitation frequency, however, has been used by many authors, ${ }^{1110}$ to accurately model other feedback mechanisms. This equation is given as

$$
S t \equiv \frac{f L}{U} \approx \frac{n-\alpha}{M+\frac{1}{\kappa}},
$$

where $\alpha$ describes the phase delay and $\kappa$ is the convection velocity of the shear layer normalized by the free stream velocity and $n=1,2,3, \ldots$ is the order of the shear layer mode. For the low subsonic speeds considered here, $\alpha=0$ was found to be appropriate, and has been used by Ma et al., ${ }^{10}$ who argued that there is no need to consider a phase delay when the convection speed is much less than the speed of sound. A convection speed of $\kappa=0.42$ was found to match the experimental measurements quite well. The typical value of $\kappa=0.5$ is the average of the free stream speed and the flow in the cavity, and does not include effects of the boundary layer which acts to retard the apparent free stream velocity.

\section{II.A. Other Instrumentation}

Varying the wind tunnel velocity provided a means to switch between the resonance conditions described in Section I. A variable speed motor driving a centrifugal blower pulled air from the lab through the inlet and test section. The motor controller was connected to a computer so that measurements as a function of tunnel velocity could be automated. The interior acoustic pressure was measured with two azimuthal rings of eight microphones. The two rings were located $125 \mathrm{~mm}$ and $200 \mathrm{~mm}$ from the end of the tunnel with the cavity opening, and the microphones were equally spaced azimuthally. Modal decomposition techniques based on the work of Bennett et al. ${ }^{12}$ were used to quantify the axial and azimuthal modes that are being excited, although only limited conclusions from the modal decomposition are presented in the present paper. The cavity and tunnel test section were constructed from clear plastic, to allow optical access for particle image velocimetry (PIV), although no PIV results are presented in this report. The microphone array and the PIV system are apparent in the photograph of the experiment, given as Figure 2. The PIV portion of this work is presented in a different conference paper by the present authors. ${ }^{13}$

\section{II.B. Measurement of Cavity Acoustic Response Function}

The acoustic response of the cavity was quantified by acoustically exciting the system with a speaker. The side of the wind tunnel opposite the cavity was removed, and a speaker was placed $0.25 \mathrm{~m}$ away from the cavity opening, with a microphone half-way in between. The cavity pressure was monitored using one of the microphones in the azimuthal array. The magnitude of the transfer function between the excitation pressure and the cavity internal pressure was calculated and the real part is shown in Figure 5 plotted as a function of Helmholtz Number,

$$
H e=\frac{2 \pi f R}{c},
$$

where $c$ is the speed of sound and $R$ is the internal radius of the cylindrical cavity. This result will be discussed in more detail in the next section.

The receptivity of the cavity to shear layer excitation was quantified using a "strength of lock-on" (SoL) parameter, as suggested by Mendelson ${ }^{14}$ and described by Yang et al. ${ }^{7}$ The parameter chosen was the amplification of the cavity pressure level above the background noise level, as defined by a linear scaling in

$\log$-log space. For each frequency, a linear fit was made to the spectral density in decibels vs the $\log _{10}$ of tunnel speed (above $4 \mathrm{~m} / \mathrm{s}$ ). The magnitude of the sound above this linear fit was designated the SoL in decibels.

\section{WEM Predictions}

A wave expansion method (WEM) code developed by Ruiz and Rice ${ }^{15}$ was used to provide insight into the acoustic response of this experiment. This WEM code was recently used by Bennett et al. ${ }^{16}$ for predicting 


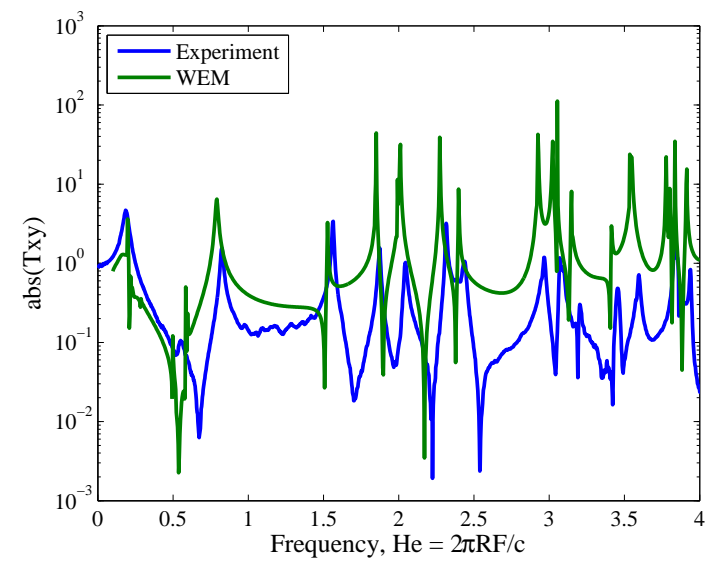

Figure 5. Cavity acoustic transfer function between the unsteady pressure at the cavity opening and the interior of the cavity. Simulation and experiment shown.
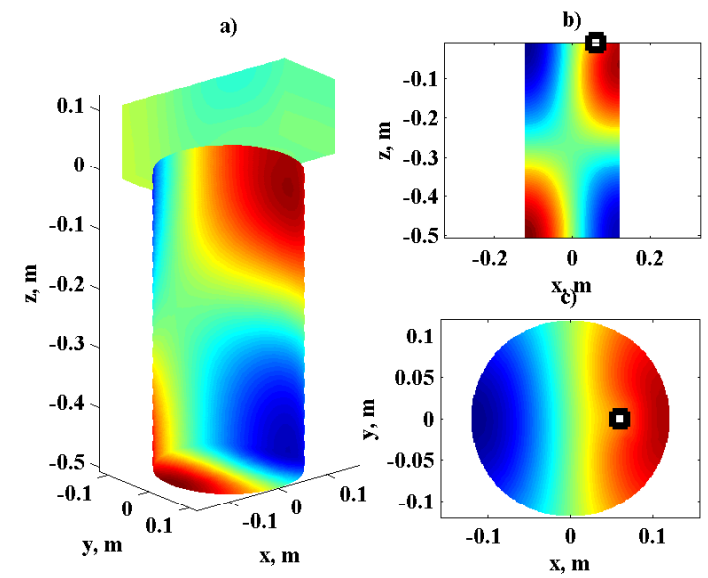

Figure 6. Internal cavity pressure predicted by WEM code. This example shows a $(1,0, .5)$ mode, occuring at $H e=2.01$. The black square denotes source location. a) Isometric view, b) Slice along streamwise centerline, c) Cavity top.

interior duct acoustic pressures, and was found to give very good agreement with analytical duct acoustic equations. The WEM code was employed to find the interior cavity pressure due to a monopole acoustic source placed at the cavity opening. The cylindrical cavity lends itself to analytical solution, but the WEM code allows for the effect of the opening to the wind tunnel to be included.

The WEM code solves for the complex pressure at all points in a domain at a single frequency. The response of the system as a function of frequency can be determined by running the code in a loop over many frequencies. A three dimensional grid encompassing the wind tunnel test section and cavity was developed, resulting in approximately 18,000 grid points. A monopole source was located at the center of the opening between the test section and the cavity. The amplification of the cavity as a function of source frequency was quantified by recording the complex pressure at the same location within the cavity as the flush mounted microphones in the experiment. An acoustic transfer function was calculated by taking the ratio of the source strength to the magnitude of the cavity pressure at this location, and the resulting function is shown in Figure 5. The cavity resonance frequencies are immediately apparent, and agree reasonably well with the acoustic measurements of the transfer function, with a small frequency offset of around $14 \mathrm{~Hz}$. Ongoing studies with the WEM code suggest that this frequency shift is due to very small differences in the dimensions of the cavity between the experiment and simulation.

The first depth mode occurs at a frequency of $H e=0.78$, with a wavelength of twice the cavity depth. The next resonance is the second depth mode at $H e=1.52$, with a wavelength equal to the cavity depth. A slightly shorter wavelength fits, however, once the first azimuthal mode cuts on, at $H e=1.84$, which is the expected circular cylinder cut-on frequency. The first "combination" mode is the combination of the first azimuthal mode and the first depth mode, which occurs at $H e=2.01$, and is shown in Figure 6 . A table of the mode types at frequencies predicted by the WEM code up to $H e=3$ is given in Table 2. The azimuthal microphone array confirmed the mode orders and frequencies in this table are the same as those occurring in the experiment, with the exception of the small shift in frequency. The first radial mode does not cut on until $\mathrm{He}=3.83$, and will not be discussed in this report.

\begin{tabular}{|c|c|c|c|c|c|c|c|}
\hline Mode (Azimuthal,Radial,Depth) & $(0,0, .5)$ & $(0,0,1)$ & $(1,0,0)$ & $(1,0, .5)$ & $(0,0,1.5)$ & $(1,0,1)$ & $(1,0,1.5)$ \\
\hline Abbreviation & D1 & D2 & AZ1 & AZ1D1 & D3 & AZ1D2 & AZ1D3 \\
\hline$H e=2 \pi f R / c$ & 0.78 & 1.52 & 1.84 & 2.01 & 2.27 & 2.39 & 2.92 \\
\hline
\end{tabular}

Table 2. Cavity resonance modes and associated frequencies and abbreviations used in this report. 


\section{Cavity Response to Grazing Flow}

The cavity opening was exposed to flow through the wind tunnel and the unsteady pressure inside the cavity was measured. The response from a single GRAS microphone, which is capable of handling the high decibel level at peak resonance conditions, is presented as spectral densities in Figures 7-9.

The use of several different cover plates for the cavity allowed the opening length and location to be changed. Specifically, three different cavity opening locations are considered, $\Delta=0,40$ and $100 \mathrm{~mm}$ with one opening length of $L=40$. Also, three different opening lengths were tested $L=40,45$ and $50 \mathrm{~mm}$ with a fixed opening location of $\Delta=0 \mathrm{~mm}$. The intention of this test scheme is to consider the effect of the two parameters separately. By changing $\Delta$ while keeping $L$ constant, the shear layer length scales are kept constant, while cavity receptivity is explored. When $\Delta$ is kept constant and $L$ is manipulated, the shear layer excitation frequencies are changed while the cavity receptivity is largely kept fixed.

\section{IV.A. Effect of Opening Location}

Figures 7-9 show the effect of varying the location of the cavity opening. All three figures are shown with the expected shear layer oscillation frequencies (denoted SL1, SL2, and SL3) overlaid as diagonal dashed lines, and the cavity resonance frequencies are overlaid as horizontal dotted lines. In all plots, the cavity receptivity is seen to be the most important parameter, with horizontal lines dominating. In many other systems, the resonance frequency exhibits some "compromise" between the shear layer frequency and the resonant response. In the present system, all cases of resonance are nearly constant in frequency.

Cavity opening centered: Figure 7 When the opening is in the center of cavity, the D1 mode dominates at all measured velocities above $32 \mathrm{~m} / \mathrm{s}$, when SL1 gets close enough to $\mathrm{He}=0.78$ to lock on. The magnitude of this resonance is high enough that significant harmonics are observed. The D2 mode and the HR mode are the second highest magnitude. The D1 mode is locked on from about $30 \mathrm{~m} / \mathrm{s}$ up to at least $52 \mathrm{~m} / \mathrm{s}$, which is the highest velocity the tunnel can achieve. This configuration is also the most receptive to the Helmholtz resonance at $8 \mathrm{~m} / \mathrm{s}$. Also apparent is a weak resonance between SL1 and D1W1, which is due to standing waves between the bottom of the cavity and the opposite tunnel wall.

Cavity opening at $R / 2$ downstream of center: Figure 8 When the opening is off center of the cavity axis, a larger variety of spectral features are apparent. The most dramatic feature occurs just above 48 $\mathrm{m} / \mathrm{s}$, where the dominant D1 mode suddenly cuts off as the free stream speed is increased. In its place, the D3 mode dominates, and the excitation by the shear layer switches from the first mode (SL1) to the second (SL2).

Cavity opening at downstream edge: Figure 9 When the opening is moved to the downstream edge of the cavity, the depth resonance modes are significantly reduced at nearly all tunnel speeds. The maximum sound level for the D1 mode is over $25 \mathrm{~dB}$ less (although this is not obvious when presented as a contour plot), and only occurs between about 30 and $40 \mathrm{~m} / \mathrm{s}$. This permits the AZ1 mode to be a maximum at around $43 \mathrm{~m} / \mathrm{s}$. The asymmetry of the excitation is observed to significantly diminish the effect of the depth mode, while the excitation of the azimuthal modes is increased.

\section{IV.B. Strength of Lock-On}

The net effect of opening location was quantified using the strength of parameter previously discussed. The results of the cavity opening location study are summarized in Figure 10, where the contour lines encircle values of SoL of $10 \mathrm{~dB}$ or more. This presentation highlights the resonance conditions. Especially noteworthy is the cut-on of the azimuthal modes AZ1 and AZ1D1 at velocities above $40 \mathrm{~m} / \mathrm{s}$ with $\Delta=0$. It is clear that the resonance lock-on for D1 is much stronger when the opening is in the center of the cavity $(\Delta=100 \mathrm{~mm})$. The central location for the opening is at a node for azimuthal modes, and would not tend to excite them. As shown in Figure 6, the downstream edge of the cavity is an anti-node for the azimuthal modes, and would be expected to excite them more efficiently. 


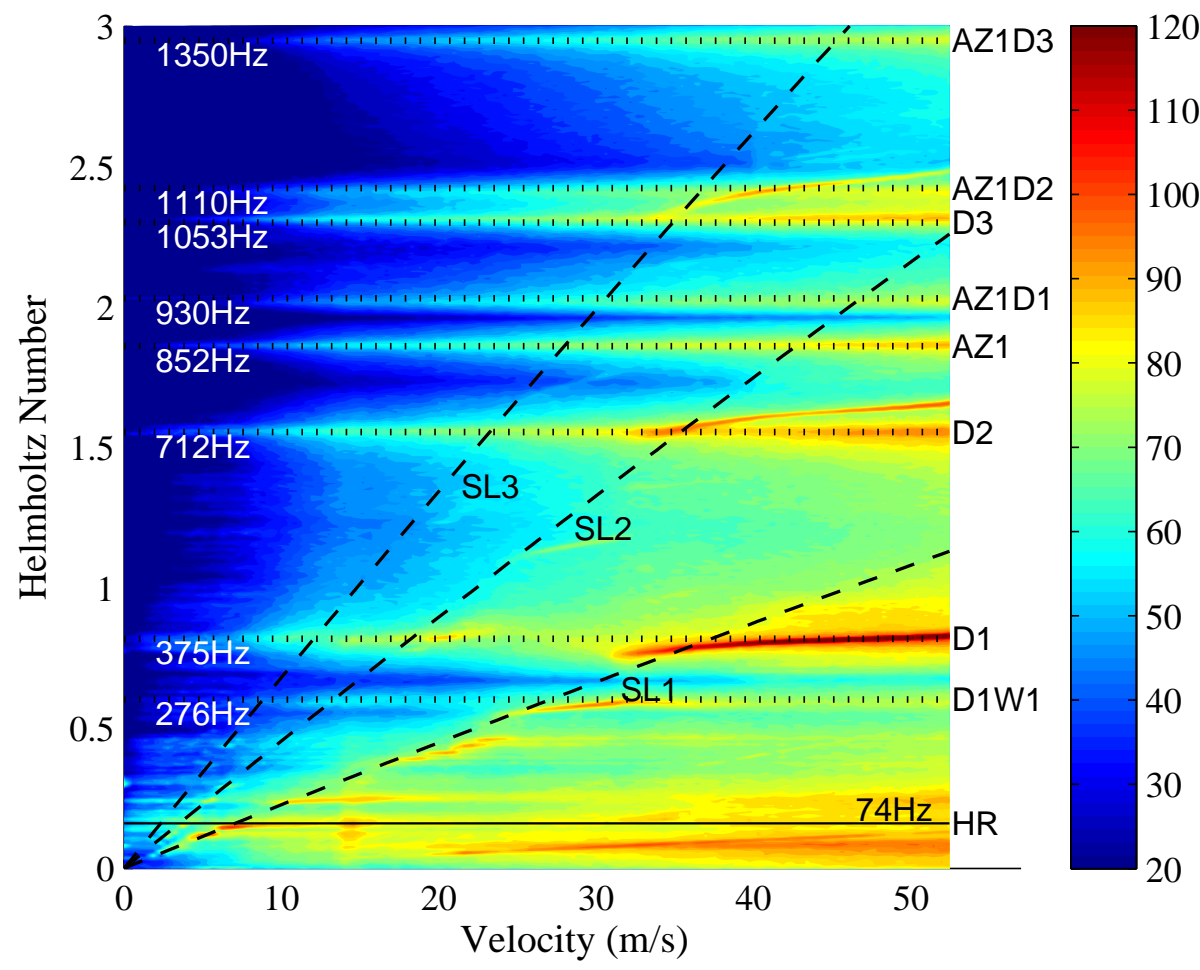

Figure 7. Pressure spectral density inside the cavity subjected to grazing flow. $L=40 \mathrm{~mm}, \Delta=100 \mathrm{~mm}$.

\section{IV.C. Effect of Opening Length}

The cavity opening length was varied from $40 \mathrm{~mm}$ to $45 \mathrm{~mm}$ to $50 \mathrm{~mm}$, with the downstream edge of the opening held even with the downstream edge of the cavity. In this case, the expected shear layer frequencies change with $L$, and are indicated by colored lines in Figure 11. Perhaps most surprising is the degree to which this figure is similar to Figure 10. Specifically, the condition of $L=50 \mathrm{~mm}, \Delta=0$ behaves in a similar way $L=40 \mathrm{~mm}, \Delta=100 \mathrm{~mm}$, although more azimuthal modes are active when $\Delta=0$.

\section{IV.D. Mode Switching}

Based on the above results, a closer look at mode switching was of interest. Figures 12 and 13 show the effect of lock-on switching from one mode to another as a function of velocity, with $L=45 \mathrm{~mm}, \Delta=0$. At $48.5 \mathrm{~m} / \mathrm{s}$, the first shear layer mode (SL1) is locked on to the first depth mode (D1), but with only a small change in tunnel velocity to $49.0 \mathrm{~m} / \mathrm{s}$, suddenly the mode AZ1D1 is excited by SL2 and dominates the spectrum. This is a significant result in the opinion of the authors, as to their knowledge only depth and longitudinal cavity resonance modes have been treated to date. There other numerous minor changes to the cavity pressure spectra between Figures 12 and 13 which are beyond the scope of the current work.

Mode oscillation occurs for some configurations and tunnel speeds. For the spectra of Figures 12 and 13, one mode dominates at each tunnel speed, and the resonance is locked-on. At $26 \mathrm{~m} / \mathrm{s}$ however, several modes share similar amplitudes and the higher frequency modes are carried on the lower, as shown in Figure 14. A spectograph analysis shows that some of these modes are unsteady in time, as seen in Figure 15. A time interval of one second is shown on the vertical axis, and the Helmholtz resonator frequency is seen to be unstable, as is the second depth mode D2 and the first azimuthal mode AZ1. 


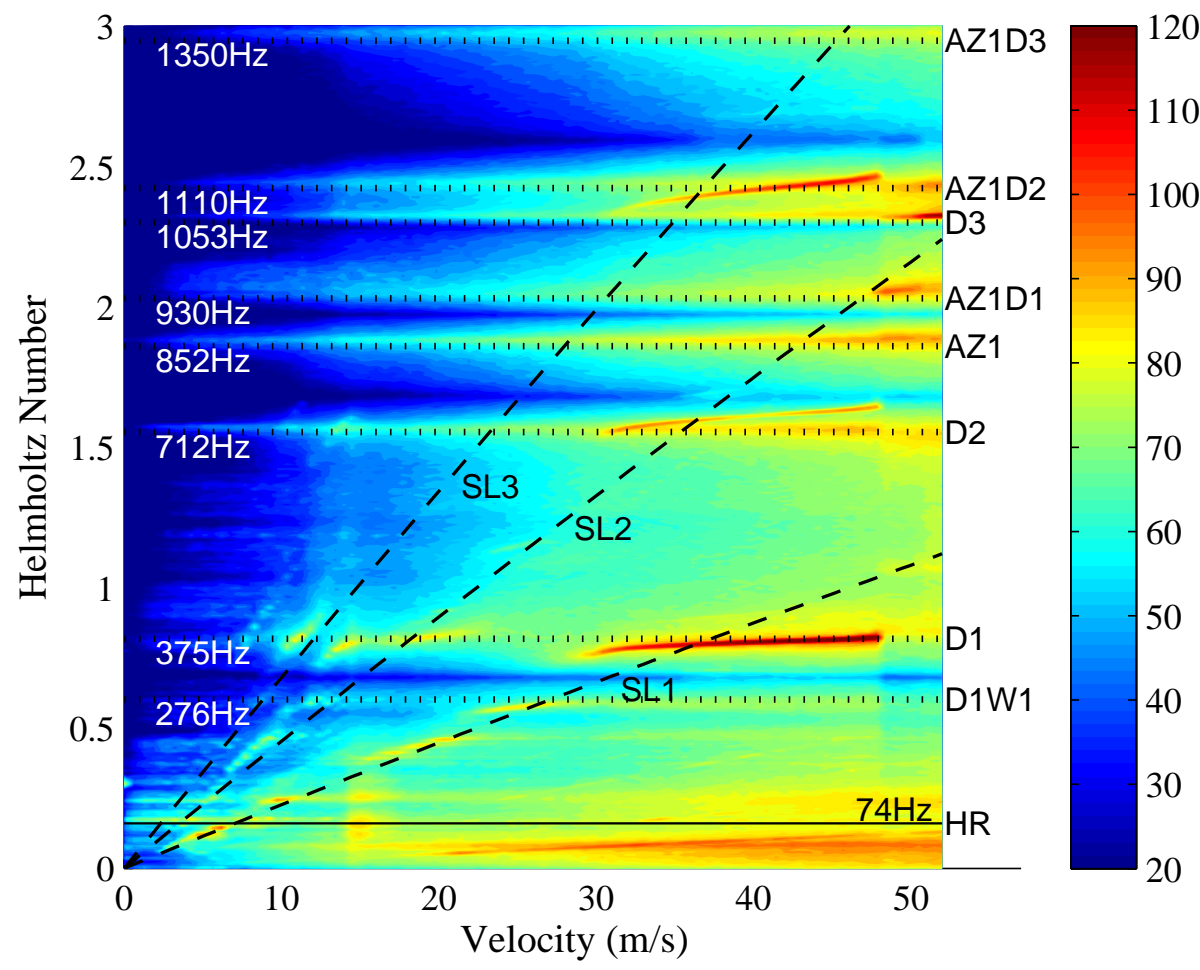

Figure 8. Pressure spectral density inside the cavity subjected to grazing flow. $L=40 \mathrm{~mm}, \Delta=40 \mathrm{~mm}$.

\section{Conclusions}

A shear layer excited cylindrical cavity has been studied, and a dominant azimuthal acoustic mode has been identified at certain free stream velocities. The effect of the cavity opening location on the internal resonance was also studied, and found to be a major factor in determining which modes were excited. Specifically, azimuthal modes were only found when the excitation was not located at the center of the cavity, which would be a node for the azimuthal mode. At low velocities, unsteady resonance was identified with continual switching between resonance conditions. Dramatic mode switching was also found at sufficiently high velocities, where dominance switched from one frequency and mode to another without any apparent oscillation. These resonance conditions should be better understood for the design of mitigation methods.

\section{Acknowledgments}

The authors would like to thank the Erasmus Mundus Master of Mechanical Engineering program for visiting scholars for financially supporting Dr. Stephens and this work at Trinity College.

\section{References}

${ }^{1}$ Langtry, R. B. and Spalart, P. R., "DES Investigation of a Baffle Device for Reducing Landing-Gear Cavity Noise," 46th AIAA Aerospace Sciences Meeting and Exhibit, Reno, Nevada, USA, 7 - 10 January 2008.

${ }^{2}$ Balasubramanian, G., Crouse, B., and Freed, D., "Numerical Simulation of Leakage Effects on Sunroof Buffeting of an Idealized Generic Vehicle," 15th AIAA/CEAS Aeroacoustics Conference, Miami, Florida, USA, 11-13 May 2009.

${ }^{3}$ Nakiboglu, G., Belfroid, S. P. C., Tonon, D., Willems, J. F. J., and Hirschberg, A., "A Paremetric Study on the Wistling of Multiple Side Branch System as a Model for Corrugated Pipes," Proceedings of the ASME 2009 Pressure Vessels and Piping Division Conference, Prague, Czech Republic, 26-30 July 2009.

${ }^{4}$ Aly, K. and Ziada, S., "Azimuthal behavior of self-excited diametral modes of internal cavities," Proceedings of the ASME 2009 Pressure Vessels and Piping Division Conference (PVP2009-77071)., Prague, Czech Republic, 2009.

${ }^{5}$ Rockwell, D. and Naudascher, E., "Review - Self-Sustaining Oscillations of Flow Past Cavities," Journal of Fluids 


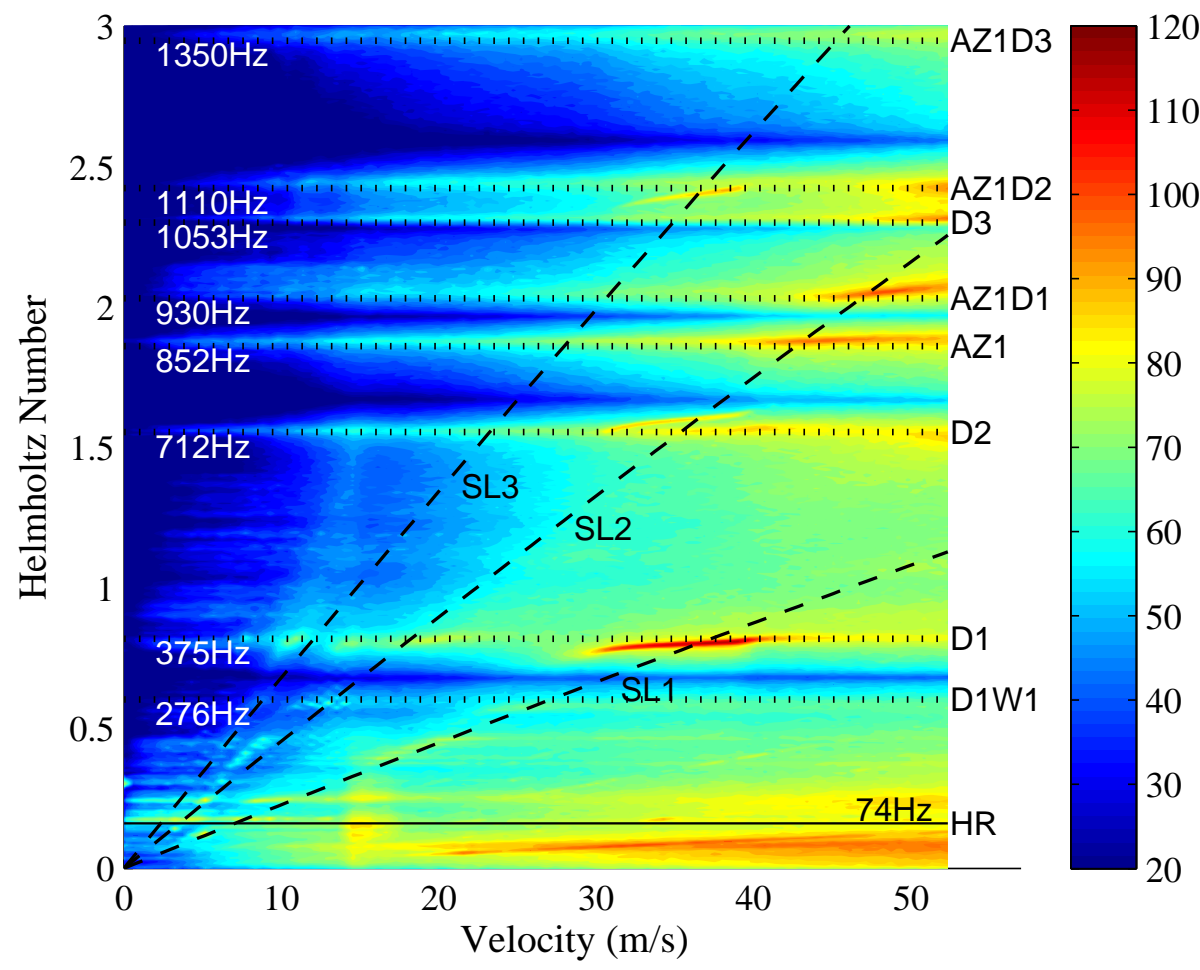

Figure 9. Pressure spectral density inside the cavity subjected to grazing flow. $L=40 \mathrm{~mm}, \Delta=0 \mathrm{~mm}$.

Engineering, Vol. 100, 1978, pp. 152-165.

${ }^{6}$ Rossiter, J. E., "Wind tunnel experiments on the flow over rectangular cavities at subsonic and transonic speeds," Reports and Memoranda No. 3438, 1964.

${ }^{7}$ Yang, Y., Rockwell, D., Cody, K. L.-F., and Pollack, M., "Generation of tones due to flow past a deep cavity: effect of streamwise length," Journal of Fluids and Structures, Vol. 25, 2009, pp. 364-388.

${ }^{8}$ Oshkai, P. and Yan, T., "Experimental investigation of coaxial side branch resonators," Journal of Fluids and Structures, Vol. 24, 2008, pp. 589-603.

${ }^{9}$ Kook, H. and Mongeau, L., "Analysis of the periodic pressure fluctuations induced by flow over a cavity," Journal of Sound and Vibration, Vol. 251, No. 5, 2002, pp. 823-846.

${ }^{10} \mathrm{Ma}$, R., Slaboch, P. E., and Morris, S. C., "Fluid mechanics of the flow-excited Helmholtz resonator," Journal of Fluid Mechanics, Vol. 623, 2009, pp. 1-26.

${ }^{11}$ Hassan, M. E., Labraga, L., and Keirsbulck, L., "Aero-acoustic oscillations inside large deep cavities," 16th Australasian Fluid Mechanics Conference, Crown Plaza, Gold Coast, Australia, 2-7 December 2007.

${ }^{12}$ Bennett, G. J., O'Reilly, C., Tapken, U., and Fitzpatrick, J., "Noise Source Location in Turbomachinery Using Coherence Based Modal Decomposition," 15th AIAA/CEAS Aeroacoustics Conference, Miami, Florida, USA, 11-13 May 2009.

${ }^{13}$ Bennett, G. J., Verdugo, F. R., and Stephens, D. B., "Shear Layer Dynamics of a Cylindrical Cavity for Different Acoustic Resonance Modes," 15th Int Symp on Applications of Laser Techniques to Fluid Mechanics, Paper Number 1727, Lisbon, Portugal, 5-8 July 2010.

${ }^{14}$ Mendelson, R. S., "Methods of measuring lock-in strength and their application to the case of flow over a cavity locking into a single sidebranch, AIAA Paper 2003-3106," 9th AIAA/CEAS Aeroacoustics Conference, Hilton Head, South Carolina, USA, 12-14 May 2003

${ }^{15}$ Ruiz, G. and Rice, H. J., "An implementation of a wave-based finite difference scheme for a 3-D acoustic problem," Journal of Sound and Vibration, Vol. 256, No. 2, 2002, pp. 373-381.

${ }^{16}$ Bennett, G. J., O'Reilly, C. J., and Liu, H., "Modelling multi-modal sound transmission from point soucres in ducts with flow using a wave-based method," 16th Congress on Sound and Vibration, Kraków, Poland, 5 - 9 July 2009. 


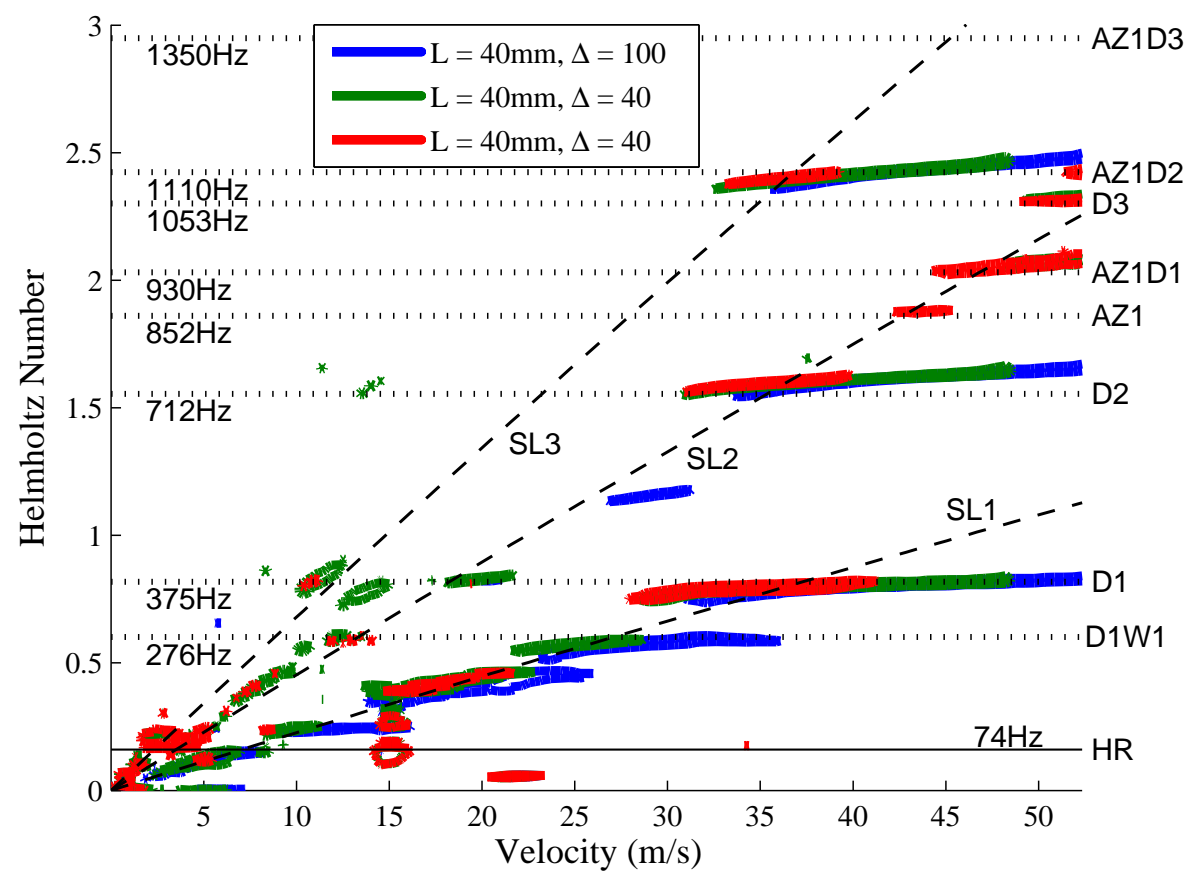

Figure 10. Strength of lock-on contours of $10 \mathrm{~dB}$ showing effect of cavity opening location. $L=40 \mathrm{~mm}$.

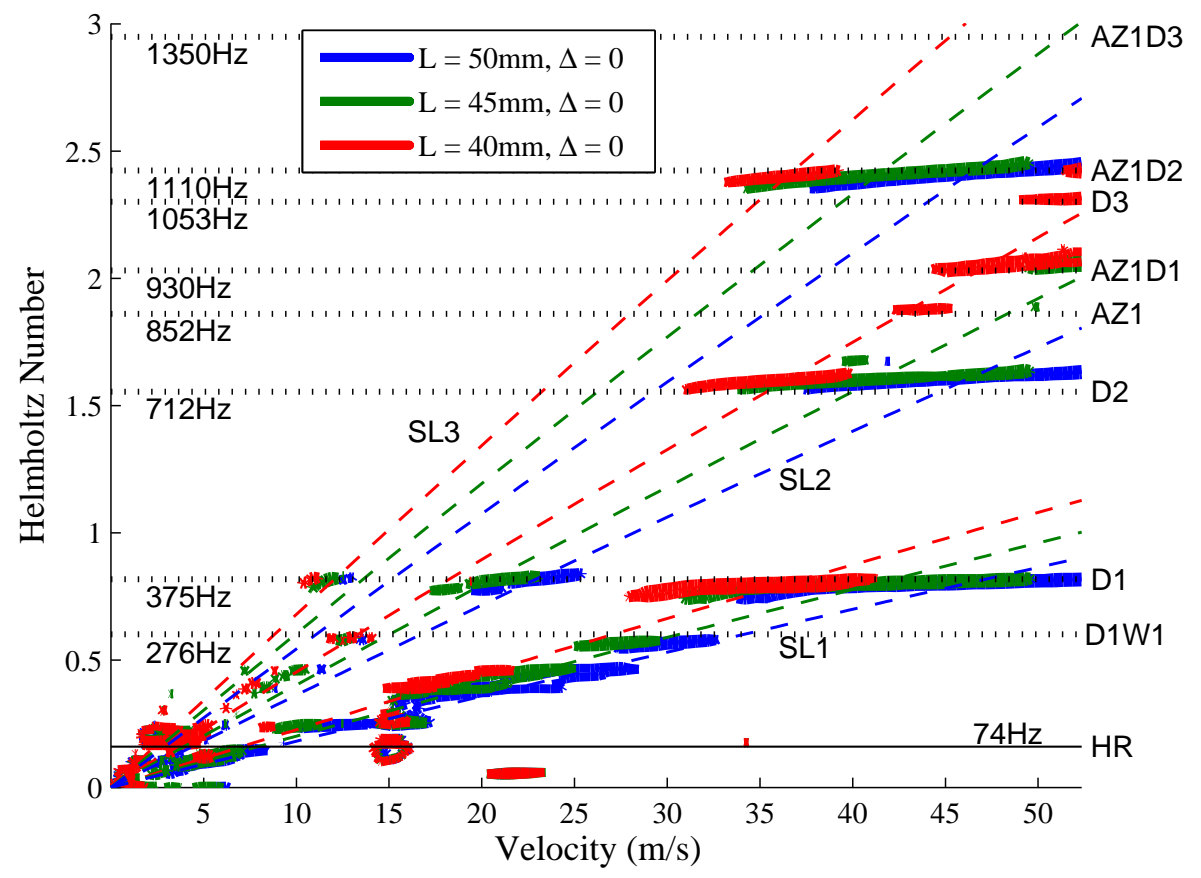

Figure 11. Strength of lock-on contours of $10 \mathrm{~dB}$ showing effect of cavity opening length. $\Delta=0$. 


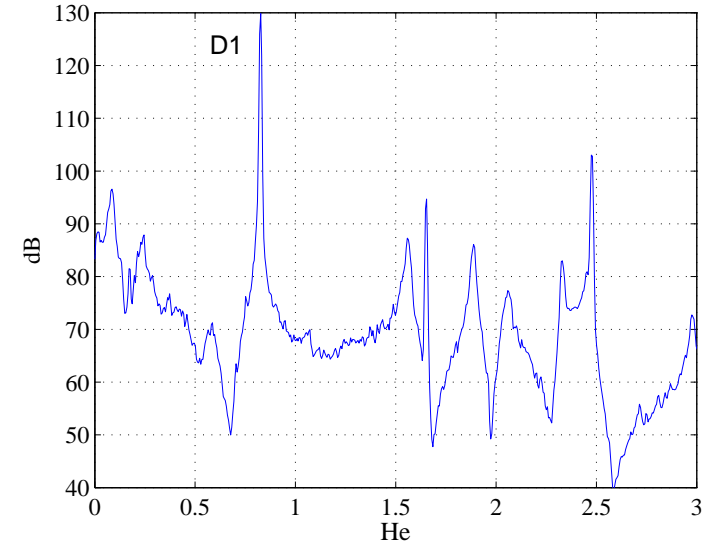

Figure 12. Internal cavity pressure with $U=48.5 \mathrm{~m} / \mathrm{s}$, $L=45 \mathrm{~mm}, \Delta=0$.

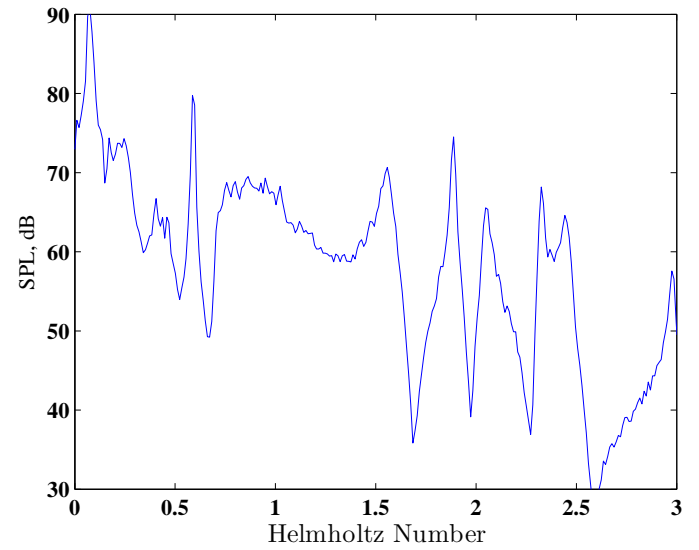

Figure 14. Internal cavity pressure spectrum measured at $26 \mathrm{~m} / \mathrm{s}$.

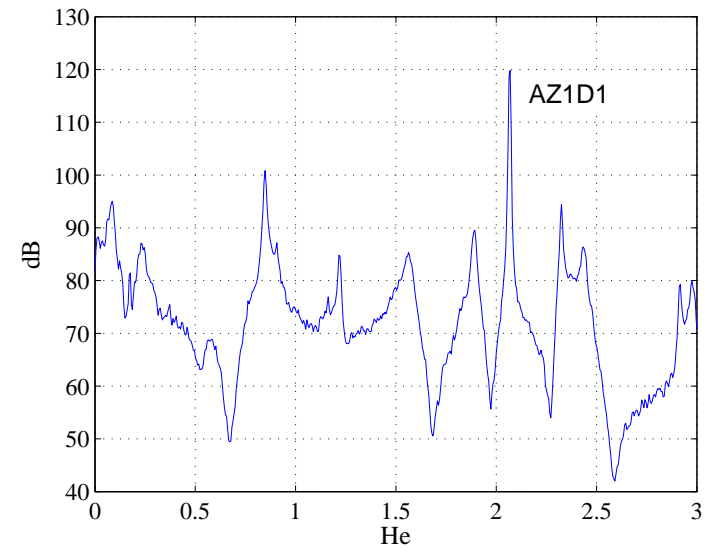

Figure 13. Internal cavity pressure with $U=49.0 \mathrm{~m} / \mathrm{s}$, $L=45 \mathrm{~mm}, \Delta=0$.

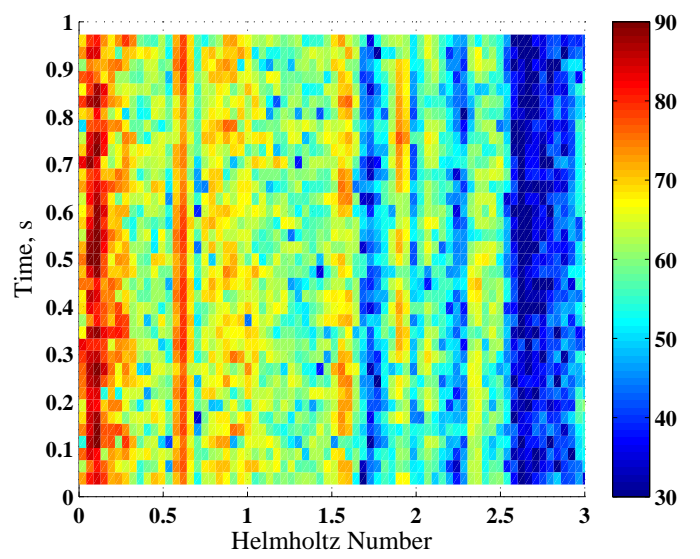

Figure 15. Spectrogram of cavity pressure at $26 \mathrm{~m} / \mathrm{s}$ showing mode switching. 